\title{
Der Unterschied der Protonenresonanzspektren von Cyclopentadienylmetallverbindungen mit Doppelkegel (Sandwich)-Struktur und lokalisierter Metall-Kohlenstoffbindung
}

\author{
Von Walter Strohmeier * und Richard M. Lemmon \\ Radiation Laboratory, University of California, Berkeley, California, USA. \\ (Z. Naturforschg. 14 a, 109-112 [1959]; eingegangen am 7. Oktober 1958)
}

\begin{abstract}
Mit Hilfe der kernmagnetischen Resonanzmethode wurden die Protonenresonanzspektren von Cyclopentadienylmetallverbindungen, welche Doppelkegel(Sandwich)-Struktur und lokalisierte MetallKohlenstoffbindungen haben, aufgenommen.

Untersucht wurden die Verbindungen: Ferrocen, 1-1'-Dimethylferrocen, Cyclopentadienylmangantricarbonyl, Methylcyclopentadienylmangantricarbonyl, Dicyclopentadienylquecksilber und Cyclopentadienyltrimethylsilicium.

Die chemical shift $\delta$ der Ringprotonen variiert von $\delta_{\mathrm{CH}}=-1,4 \mathrm{ppm}$ in Dimethylferrocen bis zu $\delta_{\mathrm{CH}}=+1,3 \mathrm{ppm}$ in Cyclopentadienyltrimethylsilicium. Die bisherigen Untersuchungen führen zu dem Schluß, daß mit zunehmendem aromatischem Charakter des Cyclopentadienringes die chemical shift nach negativeren Werten verschoben wird, während die Verbindungen mit positiveren Werten der chemical shift $\delta_{\mathrm{CH}}$ eine höhere Reaktivität gegen Maleinsäureanhydrid besitzen. Dicyclopentadienylquecksilber besitzt nur eine einzige scharfe Protonenresonanzlinie bei $\delta_{\mathrm{CH}}=+0,7 \mathrm{ppm}$, so daß es fraglich erscheint, ob diese Substanz eine, im strengen Sinne, lokalisierte Metall-Kohlenstoffbindung besitzt. Für Cyclopentadienyltrimethylsilicium wurden jedoch 2 Resonanzlinien mit dem Intensitätsverhältnis 1 : 4 für die Ringprotonen gefunden, so daß für diese Verbindung eine lokalisierte MetallKohlenstoffbindung sichergestellt ist.

Eine Analyse von Gemischen der untersuchten Verbindungen mit Hilfe der kernmagnetischen Protonenresonanzmethode ist möglich.
\end{abstract}

Das Protonenresonanzspektrum, erhalten durch kernmagnetische Resonanz, hängt unter anderem von der Elektronenverteilung in der Umgebung der Protonen ab. Es kann deshalb zur Strukturanalyse verwendet werden ${ }^{1}$, und ist in vielen Fällen auch ein einfaches Mittel zur Analyse von flüssigen Stoffgemischen. Die Lage der Protonenresonanz eines spezifischen Protons in einer bestimmten Verbindung wird durch die chemical shift $\delta$ des betreffenden Protons festgelegt. Bezugssystem ist die Lage der Protonenresonanz der Standardsubstanz Wasser, deren chemical shift definitionsgemäß $\delta_{\mathrm{H}_{2} \mathrm{O}}=0$ ist.

Nachdem in einer vorhergehenden Arbeit ${ }^{2}$ die chemical shift der Protonen in monomerem und dimerem Cyclopentadien und Methylcyclopentadien untersucht wurde, war es von Interesse, wie sich die Änderung der Elektronenverteilung im chemisch gebundenen Cyclopentadienylring in der Lage der Protonenresonanz, und somit in der chemical shift,

* Appointment supported by the International Cooperation Administration under a program administered by the $\mathrm{Na}$ tional Academy of Sciences. Derzeitige Anschrift: Physik. Chem. Institut der Universität Würzburg.

1 H. S. Gutowsky u. C. J. Hoffman, Chem. Phys. 19, 1259 [1951]. ausdrückt. Da die Elektronenverteilung im Cyclopentadienylring der Verbindungen mit Doppelkegelstruktur („aromatisierter“ Ring) eine andere als in den Cyclopentadienylverbindungen mit lokalisierter Metall-Kohlenstoffbindung (konjugierte Doppelbindungen) ist, war zu erwarten, daß das Protonenresonanzspektrum zwischen beiden Formen unterscheiden würde.

\section{Methodik}

Die Protonenresonanzspektren wurden mit einem High Resolution NMR Spectrometer V 4300 B von Varian Associates (Palo Alto, California, USA.) bei 40 Mc aufgenommen. Als Lösungsmittel kam Cyclohexan zur Verwendung, da die chemical shift aromatischer Verbindungen in $\mathrm{C}_{6} \mathrm{H}_{12}$ praktisch unabhängig von der Konzentration ist ${ }^{3}$ und von nichtaromatischen Verbindungen sich wenigstens linear mit dem Molenbruch der gelösten Substanz ändert ${ }^{4}$.

2 W. Strohmeier, E. Lombardi u. R. M. Lemmon, Z. Naturforschg. 14 a, 106 [1959].

3 P. L. Cario u. B. P. Dailey, J. Amer. Chem. Soc. 78, 3043 [1956].

4 P. L. Cario u. B. P. Dalley, Chem. Phys. 25, 1291 [1956]. 
Sämtliche Verbindungen wurden auch in Benzol oder Tetrachlorkohlenstoff gemessen, um eventuell von der $\mathrm{CH}_{2}$-Resonanz des $\mathrm{C}_{6} \mathrm{H}_{12}$ überdeckte Linien zu finden. Die Festlegung der chemical shift geschah unter Verwendung der scharfen Resonanzlinie des $\mathrm{C}_{6} \mathrm{H}_{12}$ mit $\delta_{\mathrm{CH}_{2}}=-3,9 \mathrm{ppm}^{5}$ (innerer Standard). Die Genauigkeit der gemessenen chemical shift dürfte $\pm 0,05 \mathrm{ppm}$ in bezug auf die relative Lage der einzelnen Linien zueinander, und $\pm 0,1 \mathrm{ppm}$ für die absolute Lage sein.

\section{Substanzen:}

Die Substanzen Cyclopentadienylmangantricarbonyl $\left(\mathrm{MnC}_{5} \mathrm{H}_{5}(\mathrm{CO})_{3}\right)$ und Methylcyclopentadienylmangantricarbonyl $\left(\mathrm{MnC}_{6} \mathrm{H}_{7}(\mathrm{CO})_{3}\right)$ wurden freundlicherweise von der Ethyl Corporation Detroit zur Verfügung gestellt. Ferrocen und $1-1^{\prime}$-Dimethylferrocen, für dessen Überlassung wir Herrn Prof. K. L. Rinehart, Illinois, vielmals danken, wurden durch Vakuumsublimation gereinigt. Dicyclopentadienylquecksilber $\left(\mathrm{Hg}\left(\mathrm{C}_{5} \mathrm{H}_{5}\right)_{2}\right)$ wurde nach der Lit. dargestellt ${ }^{6}$. Analyse: $\mathrm{C}=36,3 \%$; $\mathrm{H}=3,1 \%$; gefunden: $\mathrm{C}=36,44 \% ; \mathrm{H}=3,2 \%$. Das nach der Lit. ${ }^{7}$ dargestellte Cyclopentadienyltrimethylsilicium $\left(\left(\mathrm{CH}_{3}\right)_{3} \mathrm{SiC}_{5} \mathrm{H}_{5}\right)$ wurde durch Gaschromatographie (Ucon-Substrat) gereinigt. Analyse: $\mathrm{C}=69,49 \% ; \mathrm{H}=$ $10,2 \%$; gefunden: $\mathrm{C}=69,62 \% ; \mathrm{H}=10,42 \%$.

\section{Diskussion}

Protonenresonanzspektren von Cyclopentadienylmetallverbindungen wurden bereits von PIPER und $\mathrm{W}_{\text {ILKInson }}{ }^{6}$ mitgeteilt. Die Autoren fanden für Cyclopentadienylverbindungen mit Doppelkegelstruktur, wie Ferrocen, Dimethylferrocen etc., nur eine einzige Protonenresonanzlinie, was zu erwarten war, da die Protonen des Cyclopentadienylringes in einem Durchdringungskomplex in bezug auf das von außen angreifende magnetische Feld gleichwertig sein müssen. Allerdings wurde auch für Verbindungen wie Dicyclopentadienylquecksilber, für welches eine lokalisierte $\sigma$-Metall-Kohlenstoffbindung angenommen wird $^{7}$, nur eine einzige Linie mit einer breiten Schulter bei $\delta_{\mathrm{CH}} \sim 0 \mathrm{ppm}$ beobachtet ${ }^{8}$. Im Falle einer wirklich lokalisierten $\sigma$-Bindung sollten jedoch 2 Protonenresonanzlinien mit dem Intensitätsverhältnis $1: 4$ beobachtet werden ${ }^{6}$. Wir erhiel-

5 L. H. Meyer, A. Seika u. H. S. Gutowsky, J. Amer. Chem. Soc. 75, 4567 [1953].

6 T. S. Piper u. G. Wilkinson, J. Inorg. Nucl. Chem. 3, 104 [1956].

7 G. Wilkinson u. T. S. Piper, J. Inorg. Nucl. Chem. 2, 32 [1956].

8 Im folgenden sind die Werte der chemical shift von PIPeR und Wilkinson auf den Standard "Wasser" umgerechnet. Ihre Werte beziehen sich auf die Ringprotonenresonanz im Toluol mit $\delta_{\mathrm{CH}}=+1,8 \mathrm{ppm}$. Wegen des Vorzeichens der chemical shift siehe vorhergehende Arbeit 2 .

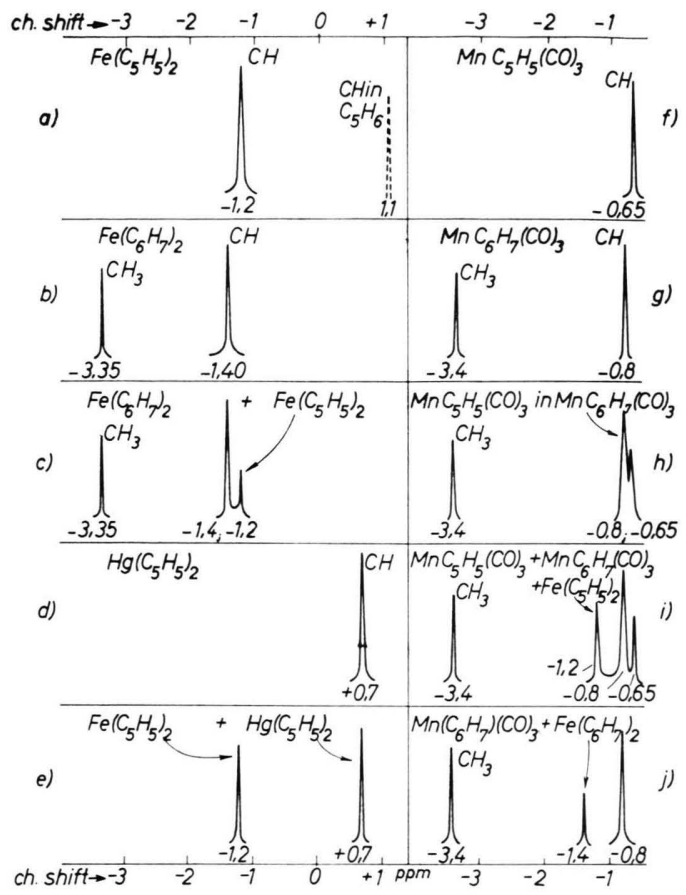

Abb. 1. Protonenresonanzspektren von Cyclopentadienylmetallverbindungen in Cyclohexan bzw. Benzol $\left(t=25^{\circ}\right)$;

a) $\mathrm{Fe}\left(\mathrm{C}_{5} \mathrm{H}_{5}\right)_{2}($ Molenbruch $x=0,03)$ in $\mathrm{C}_{6} \mathrm{H}_{12}$,

b) $\mathrm{Fe}\left(\mathrm{C}_{5} \mathrm{H}_{4} \mathrm{CH}_{3}\right)_{2}(x=0,046)$ in $\mathrm{C}_{6} \mathrm{H}_{12}$,

c) $\mathrm{Fe}\left(\mathrm{C}_{5} \mathrm{H}_{5}\right)_{2}(x=0,02)$ und $\mathrm{Fe}\left(\mathrm{C}_{5} \mathrm{H}_{4} \mathrm{CH}_{3}\right)_{2}(x=0,06)$ in $\mathrm{C}_{6} \mathrm{H}_{12}$,

d) $\mathrm{Hg}\left(\mathrm{C}_{5} \mathrm{H}_{5}\right)_{2}(x=0,07)$ in $\mathrm{C}_{6} \mathrm{H}_{6}$ (s. Anm. $\left.{ }^{10}\right)$,

e) $\mathrm{Hg}\left(\mathrm{C}_{5} \mathrm{H}_{5}\right)_{2}(x=0,07)$ und $\mathrm{Fe}\left(\mathrm{C}_{5} \mathrm{H}_{5}\right)_{2}(x=0,04)$ in $\mathrm{C}_{6} \mathrm{H}_{6}$ (s. Anm. ${ }^{10}$ ),

f) $\mathrm{Mn}\left(\mathrm{C}_{5} \mathrm{H}_{5}\right)(\mathrm{CO})_{3}(x=0,046)$ in $\mathrm{C}_{6} \mathrm{H}_{12}$,

Æ) $\mathrm{Mn}\left(\mathrm{C}_{5} \mathrm{H}_{4} \mathrm{CH}_{3}\right)(\mathrm{CO})_{3}(x=0,16)$ in $\mathrm{C}_{6} \mathrm{H}_{12}$,

h) $\mathrm{Mn}\left(\mathrm{C}_{5} \mathrm{H}_{5}\right)(\mathrm{CO})_{3}(x=0,026)$, gelöst in $\mathrm{Mn}\left(\mathrm{C}_{5} \mathrm{H}_{4} \mathrm{CH}_{3}\right)(\mathrm{CO})_{3}$,

i) $\mathrm{Mn}\left(\mathrm{C}_{5} \mathrm{H}_{5}\right)(\mathrm{CO})_{3}(x=0,05), \mathrm{Mn}\left(\mathrm{C}_{5} \mathrm{H}_{4} \mathrm{CH}_{3}\right)(\mathrm{CO})_{3}$ $(x=0,19)$ und $\mathrm{Fe}\left(\mathrm{C}_{5} \mathrm{H}_{5}\right)_{2}(x=0,05)$ in $\mathrm{C}_{6} \mathrm{H}_{12}$,

j) $\mathrm{Mn}\left(\mathrm{C}_{5} \mathrm{H}_{4} \mathrm{CH}_{3}\right)(\mathrm{CO})_{3}(x=0,32)$ und $\mathrm{Fe}\left(\mathrm{C}_{5} \mathrm{H}_{4} \mathrm{CH}_{3}\right)_{2}$ $(x=0,06)$ in $\mathrm{C}_{6} \mathrm{H}_{12}$.

ten nun, wie Abb. 1 d und Tab. 1 zeigen, bei Dicyclopentadienylquecksilber ebenfalls nur eine, allerdings scharfe, Resonanzlinie bei $\delta_{\mathrm{CH}}=+0,7 \mathrm{ppm}^{\mathbf{9}}$. Wir untersuchten deshalb noch die Verbindung Cyclopentadienyltrimethylsilicium, für welche eben-

${ }^{9}$ Die von Piper und Wilkinson beobachtete ausgewaschene Resonanzlinie des Dicyclopentadienylquecksilbers wird nach unseren Erfahrungen durch die Zersetzung des unbeständigen $\mathrm{Hg}\left(\mathrm{C}_{5} \mathrm{H}_{5}\right)_{2}$ hervorgerufen. Mehrmals frisch umkristallisiertes $\mathrm{Hg}\left(\mathrm{C}_{5} \mathrm{H}_{5}\right)_{2}$ gab jedesmal sofort nach Herstellung der Lösung eine scharfe Resonanzlinie.

10 Aus Gründen der besseren Löslichkeit wurde Benzol verwendet. Die chemical shift wurde mit Hilfe eines äußeren Standards festgelegt. 
falls eine lokalisierte Metall-Kohlenstoffbindung angenommen wird ${ }^{6,7}$. Tatsächlich konnten hier 2 Protonenresonanzlinien mit dem Intensitätsverhältnis $1: 4$ bei

$\delta_{\mathrm{CH}(\mathrm{Proton} \text { am Methylenkohlenstoffatom })}=-2,7 \mathrm{ppm}$ und $\delta_{\mathrm{CH}(\text { Protonen a. d. konjugierten Doppelbindungen) }}=+1,3 \mathrm{ppm}$

beobachtet werden (Abb. 2). Beide Resonanzlinien liegen somit ungefähr an der gleichen Stelle wie im monomeren Cyclopentadien ${ }^{2,6} \quad\left(\delta_{\mathrm{CH} 2}=-2,5\right.$ ppm und $\left.\delta_{\mathrm{CH}}=+1,1 \mathrm{ppm}\right)$. Die Aufspaltung der $\mathrm{CH}_{3}-$ Resonanzlinie im Cyclopentadienyltrimethylsilicium im Verhältnis 2: 1 (Abstand $\sim 0,1 \mathrm{ppm}$ ) deutet auf

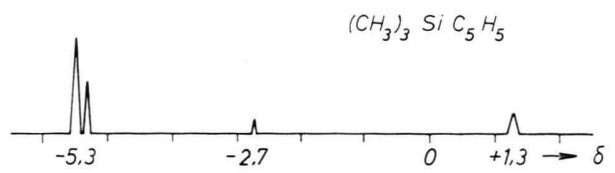

Abb. 2. Protonenresonanzspektrum von Cyclopentadienyltrimethylsilicium in $\mathrm{C}_{6} \mathrm{H}_{12}\left(t=25^{\circ}\right)$.

eine Nichtäquivalenz der $3 \mathrm{CH}_{3}$-Gruppen in bezug auf das von außen angreifende magnetische Feld hin. (Im Trimethylsiliciumchlorid wurde nur eine Resonanzlinie für die $\mathrm{CH}_{3}$-Gruppen gefunden ${ }^{2}$.)

Die Ergebnisse der kernmagnetischen Protonen- resonanz sprechen somit nur bei Cyclopentadienyltrimethylsilicium für eine wirklich lokalisierte Metall-Kohlenstoffbindung. Für die Verbindungen

$$
\begin{aligned}
& \mathrm{Hg}\left(\mathrm{C}_{5} \mathrm{H}_{5}\right)_{2}, \\
& \sigma\left(\mathrm{C}_{5} \mathrm{H}_{5}\right) \mathrm{CuP}\left(\mathrm{C}_{2} \mathrm{H}_{5}\right)_{3}, \\
& \tau\left(\mathrm{C}_{5} \mathrm{H}_{5}\right) \mathrm{Fe}(\mathrm{CO})_{2} \sigma\left(\mathrm{C}_{5} \mathrm{H}_{5}\right) \text { etc. }
\end{aligned}
$$

mit einer sogenannten $\sigma$-Metall-Kohlenstoffbindung wird von PIPER und Wilkinson angenommen, daß das Metallatom eine 1-2-Umlagerung verursacht, welche mit einer größeren Geschwindigkeit stattfindet als die zu erwartende chemical shift $(200$ bis 300 cycles/sec). Der Cyclopentadienylring kann somit als rotierend betrachtet werden, wodurch die Protonen in bezug auf das angreifende magnetische Feld gleich werden ${ }^{6}$.

In Tab. 1 sind einige typische Cyclopentadienylverbindungen nach positiver werdenden Werten der chemical shift der Ringprotonen angeordnet. Stellt man an Hand der Tab. 1 einen Zusammenhang zwischen der Lage der chemical shift der Ringprotonen und dem Bindungstyp der Metall-Cyclopentadienylringbindung her, so zeigt sich, daß nach den bisherigen Erfahrungen 3 Typen der vorwiegend ko-

\begin{tabular}{|c|c|c|c|c|c|}
\hline Verbindung & $\delta_{\mathrm{CH}_{3}}$ & $\delta_{\mathrm{CH} \text { (Ring) }}$ & $\begin{array}{l}\text { s. Anm. } \\
\text { Nr. }\end{array}$ & $\delta_{\mathrm{CH}_{2}}$ & $\begin{array}{l}\text { Reaktion mit } \\
\text { Maleinsäure- } \\
\text { anhydrid }\end{array}$ \\
\hline $\begin{array}{l}\mathrm{Fe}\left(\mathrm{C}_{5} \mathrm{H}_{4} \mathrm{CH}_{3}\right)_{2} \\
\mathrm{Fe}\left(\mathrm{C}_{5} \mathrm{H}_{5}\right)_{2} \\
\mathrm{Mn}\left(\mathrm{C}_{5} \mathrm{H}_{4} \mathrm{CH}_{3}\right)(\mathrm{CO})_{3} \\
\mathrm{Mn}\left(\mathrm{C}_{5} \mathrm{H}_{5}\right)(\mathrm{CO})_{3} \\
\pi \mathrm{C}_{5} \mathrm{H}_{5} \mathrm{Mo}(\mathrm{CO})_{3} \mathrm{C}_{2} \mathrm{H}_{5} \\
\mathrm{Ni}\left(\mathrm{C}_{5} \mathrm{H}_{5}\right) \mathrm{NO} \\
\pi \mathrm{C}_{5} \mathrm{H}_{5} \mathrm{Fe}(\mathrm{CO})_{2} \sigma \mathrm{C}_{5} \mathrm{H}_{5} \\
\pi \mathrm{C}_{5} \mathrm{H}_{5} \mathrm{Cr}(\mathrm{NO})_{2} \sigma \mathrm{C}_{5} \mathrm{H}_{5} \\
\mathrm{Hg}\left(\mathrm{C}_{5} \mathrm{H}_{5}\right)_{2} \\
\mathrm{C}_{5} \mathrm{H}_{5} \mathrm{CuP}\left(\mathrm{C}_{2} \mathrm{H}_{5}\right)_{3} \\
\mathrm{C}_{5} \mathrm{H}_{6} \\
\mathrm{C}_{5} \mathrm{H}_{5} \mathrm{CH}_{3} \\
\pi \mathrm{C}_{5} \mathrm{H}_{5} \mathrm{Fe}(\mathrm{CO})_{2} \sigma \mathrm{C}_{5} \mathrm{H}_{5} \\
\tau \mathrm{C}_{5} \mathrm{H}_{5} \mathrm{Cr}(\mathrm{NO})_{2} \sigma \mathrm{C}_{5} \mathrm{H}_{5} \\
\left(\mathrm{CH}_{3}\right)_{3} \mathrm{SiC} \mathrm{C}_{5} \mathrm{H}_{5}\end{array}$ & $\begin{array}{l}-3,35 \\
-3,4\end{array}$ & $\begin{array}{l}-1,4 \\
-1,2 \\
-0,8 \\
-0,65 \\
-0,7 \\
-0,4 \\
-0,3 \\
0,0 \\
0,7 \\
0,8 \\
1,1 \\
1,1 \\
1,2 \\
1,3 \\
1,3\end{array}$ & $\begin{array}{c}11 \\
11 \\
12 \\
6 \\
6 \\
6 \\
6 \\
6 \\
2,6 \\
2 \\
6, * * \\
6, * *\end{array}$ & $\begin{array}{l}-2,5 \\
-2,5 \\
-2,7\end{array}$ & $\begin{array}{l}- \\
- \\
- \\
- \\
- \\
- \\
+ \\
+ \\
+ \\
+ \\
+ \\
+\end{array}$ \\
\hline
\end{tabular}
valenten Metall-Cyclopentadienylringbindung zu existieren scheinen: Die $\pi$-Bindung (Doppelkegelstruk-

** chemical shift und Reaktionsfähigkeit beziehen sich auf den $\sigma$-Ring.

Tab. 1. Lage der chemical shift (in ppm) der Protonen in Cyclopentadienylverbindungen.

11 Für diese Verbindungen sind unsere Werte mit $\pm 0,1 \mathrm{ppm}$ in Übereinstimmung mit den von PIPER und Wilkinson ${ }^{6}$ angegebonen.

12 Den von Piper und Wilkinson ${ }^{6}$ angegebenen Wert von $\delta_{\mathrm{CH}}=-1,3 \mathrm{ppm}$ konnten wir nicht bestätigen. In Anbe- tracht, daß auch für $\mathrm{Mn}\left(\mathrm{C}_{5} \mathrm{H}_{4} \mathrm{CH}_{3}\right)(\mathrm{CO})_{3} \delta_{\mathrm{CH}}=-0,8 \mathrm{ppm}$ ist, erscheint uns der von uns gefundene Wert wahrscheinlicher. (Siehe auch den geringen Unterschied von $\delta_{\mathrm{CH}}$ in $\mathrm{Fe}\left(\mathrm{C}_{5} \mathrm{H}_{5}\right)_{2}$ und $\mathrm{Fe}\left(\mathrm{C}_{5} \mathrm{H}_{4} \mathrm{CH}_{3}\right)_{2}$ sowie die relative Lage der Resonanzlinien in Abb. $1 \mathrm{~h}, 1 \mathrm{i}$ und $1 \mathrm{j}$. 
tur) mit einer chemical shift der Ringprotonen von $\delta_{\mathrm{CH}}=-1,4 \mathrm{ppm}$ bis ungefähr $0 \mathrm{ppm}$, die sogenannte $\sigma$-Bindung mit einer chemical shift von $\delta_{\mathrm{CH}}>0 \mathrm{ppm}$ und die reine lokalisierte $\sigma$-Bindung wie in $\left(\mathrm{CH}_{3}\right)_{3} \mathrm{SiC}_{5} \mathrm{H}_{5}$ mit $\delta_{\mathrm{CH}}=+1,3 \mathrm{ppm}$. Ob es dazwischen kontinuierliche Übergänge gibt, kann zur Zeit nicht mit Sicherheit entschieden werden.

Tab. 1 gibt weiterhin einen Zusammenhang zwischen der chemical shift der Ringprotonen und der Reaktionsfähigkeit der Verbindungen. So reagieren die Verbindungen mit $\delta_{\mathrm{CH}}>0 \mathrm{ppm}$ ( $\sigma$-Bindung und lokalisierte Metall-Kohlenstoffbindung) mit Maleinsäureanhydrid, während in Verbindungen mit ,aro- matisiertem" Ring ( $\pi$-Bindung) die chemical shift $\delta_{\mathrm{CH}}<0 \mathrm{ppm}$ ist.

Die Unterschiede in der chemical shift der untersuchten Verbindungen ermöglichen, wie Abb. 1 c, e, $\mathrm{h}$ und $\mathrm{j}$ zeigen, eine Analyse der Stoffgemische mit Hilfe der kernmagnetischen Resonanz. In Cyclohexan wurde die chemical shift innerhalb der experimentellen Fehler und der verwendeten Molenbrüche $x$ $(x=0,02$ bis 0,4$)$ als unabhängig von der Konzentration gefunden. Cyclohexan ist somit anderen Lösungsmitteln vorzuziehen, da die Resonanzspektren, aufgenommen bei verschiedenen Konzentrationen, direkt miteinander verglichen werden können.

\title{
Kernresonanzuntersuchungen an kristallinen Flüssigkeiten: Zur Frage des molekularen Ordnungszustandes in magnetisch geordneten kristallin-flüssigen Phasen vom smektischen Typ
}

\author{
Von KarL-Heinz Weber* \\ Aus dem Physikalischen Institut der Karl-Marx-Universität Leipzig \\ (Z. Naturforschg. 14 a, 112-120 [1959]; eingegangen am 9. September 1958)
}

\begin{abstract}
In den kristallin-flüssigen, smektischen (bz-) Phasen des Azoxyphenol-di- $p$ - $n$-heptyhäthers und des Azoxybenzoesäure-di-p-äthylesters wurden bei einer Feldstärke $H_{0}=7750 \mathrm{Gauß}$ die Linienformen der magnetischen Protonenresonanzabsorption registriert und daraus die mittleren 2. Momente $\Delta H^{2}$ bestimmt. Sowohl aus der Temperaturabhängigkeit als auch aus der bei einer Drehung der Probe um eine senkrecht zum Magnetfeld gelegene Achse beobachteten Winkelabhängigkeit des 2. Moments erhält man Auskunft über die Winkelverteilung der Moleküllängsachsen im orientierenden Magnetfeld. In den smektischen Phasen beider Substanzen ist die „Mikro-Ordnung“, d. h. die Parallelorientierung der Moleküllängsachsen innerhalb kleiner Volumbereiche vollkommen, während die durch das Magnetfeld hervorgerufene „Makro-Orientierung“ der Probe unvollkommen ist; insbesondere wird am Azoxyphenol-heptyläther beim Übergang nematisch $\rightarrow$ smektisch eine Vergrößerung des MikroOrdnungsgrades $S$ und eine Verkleinerung des Makro-Ordnungsgrades $M$ beobachtet.
\end{abstract}

\section{Problemstellung}

Der kristallin-flüssige Zustand $^{1}$ ist durch die Existenz einer Vorzugsorientierung für die Längsachsen der stäbchen- oder walzenförmigen Moleküle der betreffenden organischen Verbindungen gekennzeichnet. Die Parallelorientierung der Moleküllängsachsen wird sowohl durch innere Kräfte, z. B. durch die üblichen zwischenmolekularen Wechselwirkungen und durch den Packungseffekt, als auch durch äußere Kräfte verursacht, etwa durch eine Strömung der Flüssigkeit, in dünnen Schichten der Größenordnung $10^{-1} \mathrm{~mm}$ durch Wandkräfte sowie durch ein elektrisches oder ein magnetisches Feld. Eine magnetisch geordnete kristalline Flüssigkeit verhält sich wie ein optisch einachsiger Kristall mit der optischen Achse in Feldrichtung. Jedoch führen infolge der Wärmebewegung die Moleküllängsachsen Winkelschwankungen von begrenzter Amplitude um die Vorzugsrichtung aus.

Betrachten wir speziell eine kristalline Flüssigkeit, die unter der orientierenden Wirkung eines äußeren Magnetfeldes steht, dann können zwei Fälle unterschieden werden je nachdem, ob die durch innere Kräfte verursachte Ordnung sich über die gesamte

* Neue Anschrift: VEB Vakutronik, Dresden A 21, Dornblüthstr. 14.

1 W. Kast, Z. Elektrochem. 45, 184 [1939] ; W. Kast, Angew. Chem. 67, 592 [1955]. - P. Chatelain, Bull. Soc. Franc. Minéralog. Cristallogr. 77, 323 [1954]. 